\title{
Stripping a debris disk by close stellar encounters in an open stellar cluster
}

\author{
J.-F. Lestrade ${ }^{1}$, E. Morey ${ }^{1}$, A. Lassus ${ }^{2}$, and N. Phou ${ }^{2}$ \\ 1 Observatoire de Paris/LERMA - CNRS, 61 av. de l'Observatoire, 75014 Paris, France \\ e-mail: jean-francois.lestrade@obspm.fr \\ 2 UPMC, Université Pierre et Marie Curie, 4 place Jussieu, 75005 Paris, France
}

Received 2 April 2010 / Accepted 13 June 2011

\begin{abstract}
A debris disk is a constituent of any planetary system surrounding a main sequence star. We study whether close stellar encounters can disrupt and strip a debris disk of its planetesimals in the expanding open cluster of its birth with a decreasing star number density over 100 Myr. Such stripping would affect the dust production and hence detectability of the disk. We tabulated the fractions of planetesimals stripped off during stellar flybys of miss distances between 100 and $1000 \mathrm{AU}$ and for several mass ratios of the central to passing stars. We then estimated the numbers of close stellar encounters over the lifetime of several expanding open clusters characterized by their initial star densities. We found that a standard disk, with inner and outer radii of 40 and 100 AU, suffers no loss of planetesimals over $100 \mathrm{Myr}$ around a star born in a common embedded cluster with star density $\leq 1000 \mathrm{pc}^{-3}$. In contrast, we found that such a disk is severely depleted of its planetesimals around a star born in an Orion-type cluster where the star density is $>20000 \mathrm{pc}^{-3}$. In this environment, a disk loses $>97 \%$ of its planetesimals around an M-dwarf, $>63 \%$ around a solar-type star, and $>42 \%$ around an A-dwarf, over 100 Myr. We roughly estimate that two-thirds of the stars may be born in such high star density clusters. This might explain in part why fewer debris disks are observed around lower mass stars.
\end{abstract}

Key words. circumstellar matter - Kuiper belt: general

\section{Introduction}

A debris disk surrounding a main sequence star is the collection of planetesimals (comets or asteroids) that are the leftovers from an early phase of planet formation. A debris disk is a constituent of any planetary system in the core-accretion theory of planet formation. It is the analogue of the Kuiper belt beyond Neptune, or of the asteroid belt between Mars and Jupiter, in our Solar System. The formation and evolution of debris disks and planetary systems are inter-related in theory, and can provide complementary insight into each other.

The dust content of a debris disk is the key to its observability. Small dust grains scatter the light of the central star and make the disk visible at optical wavelengths. Large grains are heated by the central star and efficiently re-radiate in the far-IR and (sub)millimeter domains where they are observed. It is thought that dust must be continually or episodicly replenished by mutual collisions between planetesimals because dust grains are removed on short timescales (a few millions years or less). Disks have to be several-fold dustier than the Kuiper belt $(\times 100)$ to be detectable with our instrumental sensitivities, even for the nearest stars. Nonetheless, bright disks are detected around $32 \pm 5 \%$ of A-type dwarfs (Su et al. 2006; Wyatt et al. 2003), $16 \pm 2.8 \%$ of solar-type dwarfs (Bryden et al. 2006; Trilling et al. 2008; Najita \& Williams 2005) and $\leq 5 \%$ of M-type dwarfs (Lestrade et al. 2006, 2009; Gautier et al. 2007) according to searches for cold dust at $\lambda=70 \mu \mathrm{m}$ by Spitzer and at (sub)millimeter wavelengths by radiotelescopes. Debris disks were recently reviewed by Wyatt (2008).
The mechanisms responsible for enhanced collisional activity and for grinding planetesimals to dust in debris disks remain unclear. The statistics of disk detections just recalled cannot be accurately interpreted unless these mechanisms are identified. It has been proposed that unseen planets dynamically stir the disk and generate copious amount of dust that evolve under gravity, radiation pressure, P-R, and stellar-wind drags (Moro-Martín \& Malhotra 2002; Wyatt 2003; Mustill \& Wyatt 2009; Kennedy \& Wyatt 2010). Steady-state equilibrium between collisional cascades of planetesimals and dust removal processes can explain how disks fade with age (Dominik \& Decin 2003). Orbit resonance crossing of giant planets can trigger abrupt showers of planetesimals in the system, severely depleting the disk and limiting its detectability (Gomes et al. 2005; Morbidelli et al. 2005; Tsiganis et al. 2005). However, the distribution of disk fractional luminosities with star ages is inconsistent with the idea that this mechanism is common among debris disks (Booth et al. 2010). Finally, distant icy-planets can successively form in waves outwards in the disk and generate dust rings (Kenyon \& Bromley 2002).

In this paper, we study whether stellar flybys during the first $100 \mathrm{Myr}$ of the lifetime of a star, while it is still in the expanding open cluster of its birth, can significantly deplete a disk of its planetesimals, affecting its dust production and therefore detectability. Stellar flybys have been invoked to explain the high eccentricity orbits of some Kuiper belt objects such as Sedna (Kenyon \& Bromley 2004), the dynamics of planetary systems (Malmberg et al. 2007; Spurzem et al. 2009), and the structures of debris disks (Larwood 1997; Mouillet et al. 1997; Kalas et al. 2000; Kobayashi \& Ida 2001). Impacts of stellar flybys during 
the first few million years have also been studied in the context of the evolution of protoplanetary disks and planet formation (Bonnell et al. 2001; Olczack et al. 2009).

For our study, we did not resort to a full $N$-body integration of an expanding cluster with stars surrounded by disks of planetesimals. We instead divided the problem into parts by first estimating the fraction of planetesimals stripped off a disk by the passage of a star moving on a parabolic trajectory in Sect. 2, and second by estimating the probability of close stellar encounters in an expanding cluster in Sect. 3. Finally, we discuss our results in Sect. 4.

\section{Stripping a debris disk by stellar flybys}

In our model, the central and the passing stars are considered to be point masses, named $m_{\mathrm{c}}$ and $m_{\mathrm{p}}$, respectively. The disk is made of $10^{4}$ massless planetesimals randomly distributed, radially and azimutally, with the planetesimal surface number density $\Sigma(a)=\Sigma_{0}\left(a / a_{0}\right)^{-1.5}$. Hence, the disk is not self-gravitating, i.e., the orbit of each planetesimal is computed under the sole gravitational attractions of $m_{\mathrm{c}}$ and $m_{\mathrm{p}}$ in the restricted three-body problem.

In our simulations, we considered two sizes for the disk: a standard configuration with inner and outer radii of 40 and $100 \mathrm{AU}$, and a more compact configuration with respective radii 10 and $40 \mathrm{AU}$, at the start of the computation. These characteristic radii are based on limited observations at present. Protoplanetary transition disks have inner radii ranging from 15 to $73 \mathrm{AU}$ and outer radii from 30 to $135 \mathrm{AU}$, as measured by the SMA (Andrews et al. 2011). More mature debris disks around main sequence stars have radii determined by means of either imaging or the fitting of their SED, and comprised between $\sim 10$ AU and 300 AU (e.g., Dent et al. 2000; Greaves et al. 2005; Liseau et al. 2008; Corder et al. 2009; Marshall et al. 2011). In addition, disk size may depend on the central star mass; for example, the disk surrounding the M-star AU Mic is about three times smaller than the disk around the A-star $\beta$ Pic of the same age (12 Myr) (Augereau \& Beust 2006; Augereau et al. 2001). However, a correlation between disk size and star mass has not yet been established because of a lack of sufficient data. Current surveys with Herschel in the far-IR (Matthews et al. 2010; Eiroa et al. 2010) will provide new and important constraints. We adopted the two disk configurations, standard and compact, in our simulations to help us prepare for any revisions of disk sizes in the future.

Our first suite of simulations was conducted in a threedimensional (3D) coordinate system where the disk is confined to the $x-y$ plane but the parabolic orbit of the passing star can be in an inclined plane. The starting position of the passing star is chosen to be at a large distance ( $>1000 \mathrm{AU})$ to ensure that the disk is not perturbed by an impulsive force at the start of the computation. The initial velocity for the passing star is set so that, at the closest approach, it reaches a given miss distance while on a parabolic trajectory. The miss distances were set to be between $100 \mathrm{AU}$ and $1000 \mathrm{AU}$, and the resulting maximum velocities of the passing star at closest approaches were between 3 and $12 \mathrm{~km} \mathrm{~s}^{-1}$. This maximum velocity is related to the miss distance because of the parabolic nature of the orbit.

The equation of motion of a massless particle under the gravitational attractions of the central and passing stars were written in a coordinate system where the central star is at the origin. These equations were integrated numerically using the fourth order Runge-Kutta algorithm described in Press et al. (1992). The numerical accuracy of our implementation of this algorithm was tested by computing the total energy of a test star, i.e., one not gravitationally interacting with the disk, set on a high eccentricity orbit with a small semi-major axis to produce the highest acceleration in the system. We set the time step for our integration so that the total energy of this test star was conserved to an accuracy lower than $0.1 \%$. We also extensively compared our Runge-Kutta results to the ones that we obtained first with the computationally expensive Euler method.

The dynamical evolution of our system is simulated over a few thousand years, long enough for the passing star to reach the closest approach and move away to a large distance. Our simulation is exemplified in Fig. 1 for prograde and coplanar motions of the disk and passing star. This example shows that the disk is first slowly perturbed and then significantly disrupted at closest approach where the tidal forces reach a maximum. Finally the disk settles in to its new configuration with bound planetesimals on elliptical orbits, while other planetesimals are either captured by the passing star or ejected on unbound orbits. We checked that the fractions of stripped planetesimals (captured + ejected) are accurately estimated for a disk confined to the $x-y$ plane and accurately represented by $10^{4}$ particles in comparing to simulations with larger numbers of particles.

Our simulations were carried out by varying the miss distance from $100 \mathrm{AU}$ to $1000 \mathrm{AU}$, the sense of circulation between the disk and the passing star (prograde, retrograde), the inclination $i$ of the orbital plane of the passing star $\left(0^{\circ}, 30^{\circ}, 45^{\circ}\right.$, $\left.90^{\circ}\right)$, and the mass radtio $m_{\mathrm{p}} / m_{\mathrm{c}}(20,10,4,2,1,0.5,0.25,0.1)$. We found that all stellar flybys with retrograde motions are inefficient leading to very low fractions of stripped planetesimals. This is because they do not move in concert with the passing star and are not gravitationally perturbed for a sufficiently long period of time, as also noted by other authors in similar circumstances (Toomre \& Toomre 1972; Beust \& Dutrey 2006; Reche et al. 2009). All flybys with prograde motion and inclination $i \geq 45^{\circ}$ are also inefficient. Only flybys with prograde motion and inclination $i<45^{\circ}$ lead to significant stripping whose magnitude is quantified by Figs. 2 and 3. These figures show the fraction of stripped planetesimals as a function of the miss distance, for the mass ratios $m_{\mathrm{p}} / m_{\mathrm{c}}$ mentioned above, for the standard and compact disks, and for inclination $i<45^{\circ}$ of the orbital plane of the passing star. Clearly, by comparing Figs. 2 and 3, a compact disk with inner and outer radii of 10 and $40 \mathrm{AU}$ is much less severely depleted of its planetesimals than the more extended one.

To check our simulations, we duplicated the results of Larwood \& Kalas (2001) for the debris disk around $\beta$ Pic perturbed by a passing star. We found excellent agreement with their Table 1 for the numbers of particles unbound and captured by the passing star, for all ratios $m_{\mathrm{p}} / m_{\mathrm{c}}$ and inclinations. We also compared our results with the study of stripping effects in the Kuiper belt when the Solar System was in its birth aggregate performed by Adams \& Laughlin (2001). They estimate that $40 \%$ of the Kuiper belt objets with semi-major axis between 30 and $70 \mathrm{AU}$ are removed from the Solar System in an encounter with a 1 solar mass star at the miss distance $\sim 200 \mathrm{AU} \sim 350 / \sqrt{\pi} \mathrm{AU}$ by using their fiducial cross-section $\sigma \sim(350 \mathrm{AU})^{2}$. This fraction is higher than our prediction of $13 \%$ in Fig. 2. We ascribe this difference to their model which also includes the scattering of high eccentricity planetesimals by Neptune that is not considered in our model.

Finally, we studied how the initial conditions in the disk affect the outcome of a flyby since the disk can be initially excited by the dynamical perturbations of a previous flyby. We run a new suite of simulations in which planetesimals were set on eccentric 

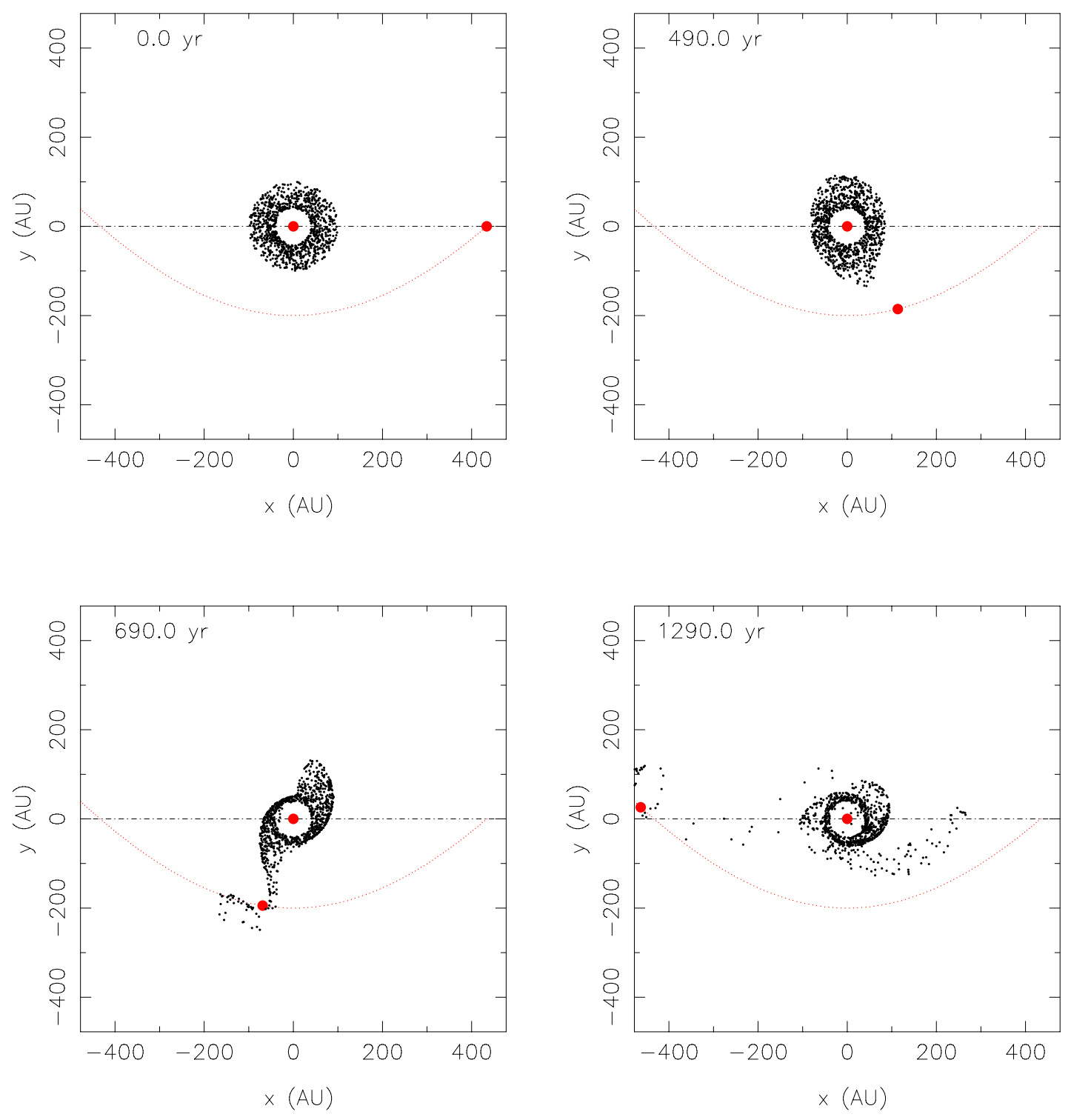

Fig. 1. Stellar flyby. The debris disk is non-self-gravitating, and has inner and outer radii of 40 and 100 AU at the start of the simulation. The central star is at the origin of the coordinate system. The trajectory of the passing star is parabolic, coplanar and prograde with respect to the disk. The mass ratio of the two stars is unity. At the closest approach, the miss distance is $200 \mathrm{AU}$ and the resulting maximum velocity is $4.2 \mathrm{~km} \mathrm{~s}^{-1}$. The fraction of planetesimals stripped off the disk and lost by the system is $13 \%$ during this close stellar encounter.

and inclined, instead of circular, orbits. Their eccentricities, inclinations, and semi-major axes $a$ were randomly distributed between 0.0 and $0.95, \pm 30^{\circ}$, and 40 and 100 AU, respectively, at the start of the simulation. The planetesimal surface number density $\Sigma(a)$ was the same power-law as given above and $10^{5}$ particles were used to represent this three-dimensional (3D) disk. These new simulations have yielded fractions of stripped planetesimals that are somewhat larger than for a disk with no initial excitation, but not exceedingly larger. These new fractions are the points connected by dotted lines in Figs. 2 and 3. To appreciate the differences, we also present a subset of these points in Table 1 for a standard disk, miss distance of 200 AU and mass ratios $m_{\mathrm{p}} / m_{\mathrm{c}}=4,2,1,0.5$, and 0.25 , which are representative examples. We estimated that the final depletions after $100 \mathrm{Myr}$ between the two models can change by at most a factor of two depending on the exact history of encounters. Such a change is not important for the assessment of the impact of depletion on the dust production of a disk; only a depletion factor greater than
Table 1. Stellar flybys of miss distance 200 AU for two initial disk excitations *

\begin{tabular}{|c|c|c|}
\hline \multirow[t]{2}{*}{$m_{\mathrm{p}} / m_{\mathrm{c}}$} & \multicolumn{2}{|c|}{ Fractions of stripped planetesimals } \\
\hline & no excitation & excitation \\
\hline 4 & $47 \%$ & $63 \%$ \\
\hline 2 & $27 \%$ & $42 \%$ \\
\hline 1 & $12 \%$ & $21 \%$ \\
\hline 0.5 & $5 \%$ & $12 \%$ \\
\hline 0.25 & $1.2 \%$ & $6.2 \%$ \\
\hline
\end{tabular}

Notes. ${ }^{(*)}$ Standard size disk.

ten is meaningful for the disk detectability. For the remaining analysis, we adopted the fractions given in Figs. 2 and 3 for a disk with no initial excitation. 


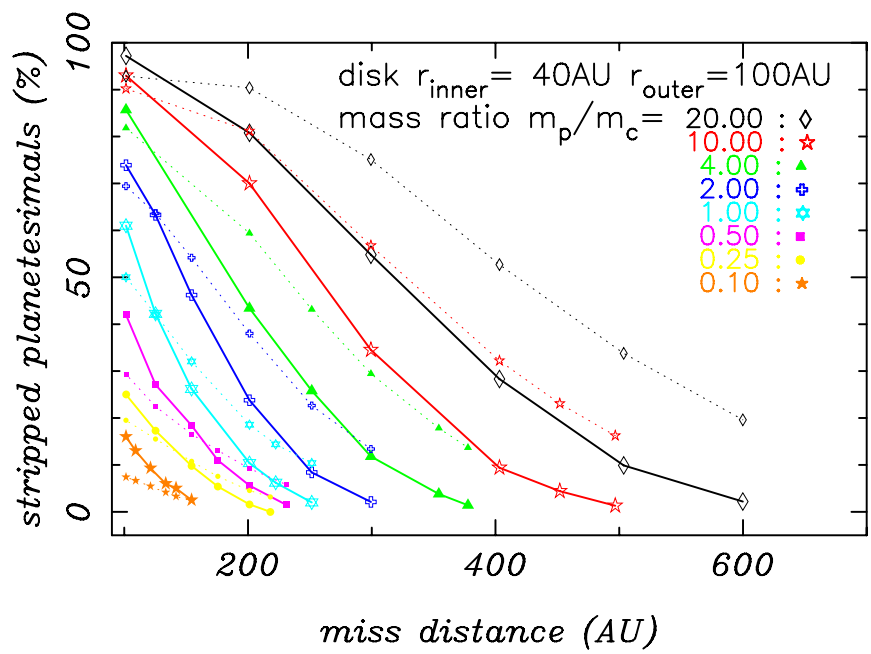

Fig. 2. Fractions of planetesimals stripped off a disk during stellar flybys at various miss distances and for various color-coded mass ratios $m_{\mathrm{p}} / m_{\mathrm{c}}$ of the passing to central stars. The motions of the disk and of the passing star are prograde and their orbital planes are coplanar. The inner and outer radii of the disk are 40 and $100 \mathrm{AU}$, respectively, at the start of the simulation. Thick lines connect the points computed for a disk with planetesimals initially set on circular orbits. Dotted lines connect the points computed for a dynamically excited disk with orbits of planetesimals initially set with random eccentricities and inclinations (see details at end of Sect. 2).

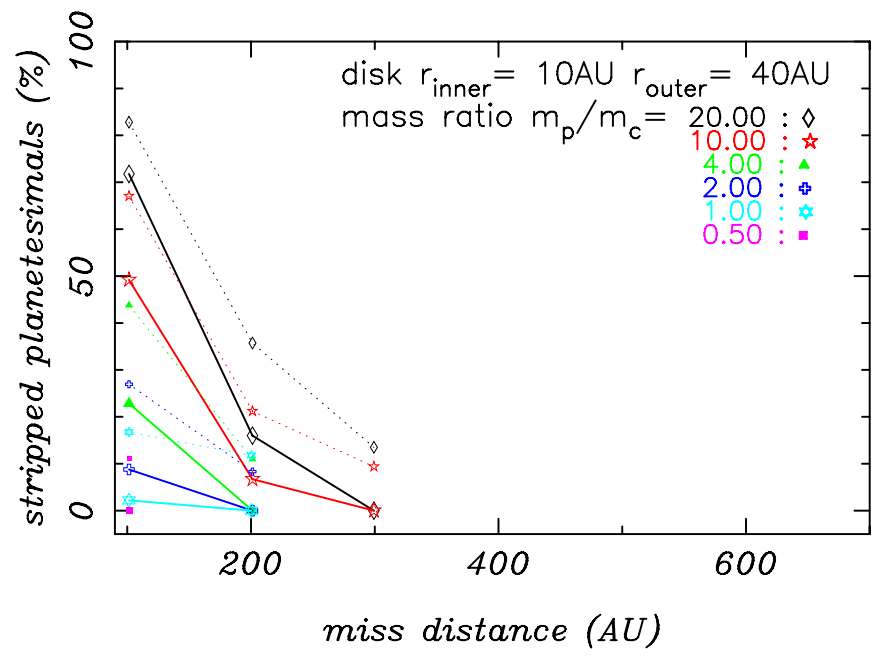

Fig. 3. Same as Fig. 2 but for a compact disk with inner and outer radii of 10 and $40 \mathrm{AU}$.

\section{Close stellar encounters in an expanding open cluster}

\subsection{Number of stellar encounters over the cluster lifetime}

We do not resort to an $N$-body calculation to estimate the frequencies of encounters between stars in an open cluster. We instead use the kinetic theory complemented by gravitational focusing. In this framework, the encounter time $t_{\text {enc }}$ can be estimated analytically by rederiving Eqs. (8)-(122) of Binney \& Tremaine (1987, p. 539), which is valid for two stars of identical masses, for the more general case of a central star and a passing star of masses $m_{\mathrm{c}}$ and $m_{\mathrm{p}}$ moving in a field with uniform star number density $n$. The corresponding encounter rate at miss distance $d<d_{\text {enc }}$ is

$\frac{1}{t_{\mathrm{enc}}}=4 \sqrt{\pi} n \sigma d_{\mathrm{enc}}^{2}+\frac{2 \sqrt{\pi} G\left(m_{\mathrm{c}}+m_{\mathrm{p}}\right) n d_{\mathrm{enc}}}{\sigma}$,
Table 2. Stellar mass distribution of passing stars.

\begin{tabular}{lcr}
\hline \hline Spectral type $m_{\mathrm{p}}$ & $\begin{array}{c}\text { Mass range } \\
\left(M_{\odot}\right)\end{array}$ & Fraction $f_{\mathrm{p}}$ \\
\hline M8-M5 & $0.10-0.21$ & $43.0 \%$ \\
M4-M0 & $0.21-0.47$ & $31.5 \%$ \\
K8-K0 & $0.47-0.80$ & $12.4 \%$ \\
G8-G0-F0 & $0.80-1.70$ & $8.5 \%$ \\
A8-A0 & $1.70-3.20$ & $2.7 \%$ \\
B8-B5 & $3.20-6.50$ & $1.3 \%$ \\
\hline
\end{tabular}

where $\sigma$ is the dispersion of the Maxwellian distribution in the stellar velocities of the cluster, and $G$ is the gravitational constant. The first term is the collision rate inferred from the simple kinetic theory of a star moving at the mean velocity $4 \sigma / \sqrt{\pi}$ (mean velocity of a Maxwellian with dispersion $\sigma$ ). This expression is based on the volume swept by the cross-section $\pi d_{\mathrm{enc}}^{2}$ at this mean velocity during $t_{\mathrm{enc}}$. The second term is gravitational focusing to account for the true orbits of the two stars. In convenient units, the total encounter rate is

$$
\begin{aligned}
& \frac{1}{t_{\mathrm{enc}}}\left(\mathrm{yr}^{-1}\right)=1.9 \times 10^{-8}\left(\frac{n}{1000 \mathrm{pc}^{-3}}\right)\left(\frac{\sigma}{1 \mathrm{~km} \mathrm{~s}^{-1}}\right)\left(\frac{d_{\mathrm{enc}}}{100 \mathrm{AU}}\right)^{2} \\
& \quad+8.8 \times 10^{-9}\left(\frac{m_{\mathrm{c}}+m_{\mathrm{p}}}{1 M_{\odot}}\right)\left(\frac{n}{1000 \mathrm{pc}^{-3}}\right)\left(\frac{d_{\mathrm{enc}}}{100 \mathrm{AU}}\right)\left(\frac{1 \mathrm{~km} \mathrm{~s}}{\sigma}\right) .
\end{aligned}
$$

For our model, we assume that the star number density $n$ is a linear function of time $t$. In our notation, this is

$n=n_{0} f_{\mathrm{p}}-\left(n_{0}-n_{1}\right) f_{\mathrm{p}}\left(t / t_{\mathrm{cl}}\right)$,

where $f_{\mathrm{p}}$ is the stellar fraction for each spectral type range listed in Table 2. With this linear expression of time, the total star number density in the cluster decreases from $n_{0}$ at $t=0$ to $n_{1}=0.1 \mathrm{pc}^{-3}$ at $t=t_{\mathrm{cl}}$, where $t_{\mathrm{cl}}$ is the cluster lifetime, typically 100 Myr (Lada \& Lada 2003). The final value of $0.1 \mathrm{pc}^{-3}$ is the field star density as measured in the solar neighbourhood as well as in relatively old open clusters (Abt 2009). The linear dependence adopted is based on the study of the dynamical evolution of the Pleiades over 1 Gyr by Converse \& Stahler (2011). They found that the central surface density $\left(\mathrm{pc}^{-2}\right)$ of the cluster falls linearly with time at a rate such that the initial density drops by tenfold in $200 \mathrm{Myr}$, and then falls also linearly but at a slower rate until $1 \mathrm{Gyr}$ when the cluster is totally dispersed. They also show that the core radius is almost constant during the first $125 \mathrm{Myr}$ such that the linear dependence of the surface number density $\left(\mathrm{pc}^{-2}\right)$ transfers to the volume density $\left(\mathrm{pc}^{-3}\right)$. We also note that in a similar way, Kaib \& Quinn (2008) use this a priori time dependence for their sudy of the formation of the Oort cloud in an open cluster environment.

The number of stellar encounters of miss distances $d<d_{\text {enc }}$ can be tracked by the phase (cycle)

$\phi\left(d<d_{\mathrm{enc}}\right)=\int_{0}^{t_{\mathrm{cl}}} 1 / t_{\mathrm{enc}} \mathrm{d} t$.

Stellar flybys of miss distances $<d_{\text {enc }}$, mean velocity $4 \sigma / \sqrt{\pi}$, and in the environment of varying star density $n$ of Eq. (3), occur successively at each integer value taken by this phase.

We checked our algorithm in predicting the close stellar encounter rate $\Gamma$ modeled by Proszkow \& Adams (2009) in their $N$-body simulations of stars in an embedded cluster. Their power 
law $\Gamma=\Gamma_{0} \times(b / 1000 \mathrm{AU})^{\gamma}$ corresponds to the number of close stellar encounters of miss distances $<b$ per star per Myr. With our model, we estimated the time $T_{1000 \mathrm{AU}}$ required for the first stellar flyby at $d<1000 \mathrm{AU}$ to occur by making $\phi(d<1000 \mathrm{AU})=\int_{0}^{T_{1000 \mathrm{AU}}} 1 / t_{\mathrm{enc}} \mathrm{d} t=1$ with the constant stellar density $n=1000$ stars pc $^{-3}$ in Eq. (2). In a similar way, we also estimated $T_{500 \mathrm{AU}}$ for $d<500 \mathrm{AU}$. With these two values $T_{1000 \mathrm{AU}}$ and $T_{500 \mathrm{AU}}$, we determined $\Gamma_{0}=0.4$ flybys $(b<1000 \mathrm{AU})$ per star per Myr and $\gamma=1.5$ if $\sigma=1 \mathrm{~km} \mathrm{~s}^{-1}$, and determined $\Gamma_{0}=1$ flyby $(b<1000 \mathrm{AU})$ per star per Myr and $\gamma=1.9$ if $\sigma=5 \mathrm{~km} \mathrm{~s}^{-1}$. These values of $\Gamma_{0}$ and $\gamma$ are consistent with those in Proszkow \& Adams (2009). As an additional test of our algorithm, we also predicted for the Sun that a close stellar encounter of $0.5 \mathrm{pc}$ occurs once every $1.2 \mathrm{Myr}$ in the present-day star field ( $n=$ const. $=0.1 \mathrm{star} \mathrm{pc}^{-3}$ in Eq. (2)). This prediction is indeed the frequency determined by GarciaSanchez et al. (2001).

\subsection{Depletion of debris disks}

We turn now to our inital problem and apply Eqs. (2)-(4) to estimate the fraction of planetesimals left in a debris disk after close stellar encounters have occurred for the first 100 Myrs of its lifetime. For this estimate, we need to determine the miss distance for which six stellar flybys occur during this period, hence Eq. (4) becomes $\phi\left(d<d_{\mathrm{enc}}\right)=\int_{0}^{t_{\mathrm{cl}}} 1 / t_{\mathrm{enc}} \mathrm{d} t=6$ with $t_{\mathrm{cl}}=$ 100 Myr. We demonstrate below why six flybys are required. We solve numerically this equation for $d_{\text {enc }}$ and use its value in Figs. 2 or 3 to estimate the fraction of stripped planetesimals. This fraction is a lower limit, i.e., more planetesimals are ejected in reality, for two reasons. First, the miss distance of an encounter tracked by Eq. (4) is $d_{\text {enc }}$ or smaller, and closer encounters more severely deplete a disk. Second, stellar flybys at larger miss distances that are tracked by $\phi\left(d<d_{\text {enc }}\right)=12,18,24, \ldots$ in our algorithm are ignored in this work, although these flybys do slightly increase the fraction of stripped planetesimals. This ensures that our final conclusion is conservative but not overly. In practice, we solve numerically the equation $\phi\left(d<d_{\text {enc }}\right)=6$ above for each stellar spectral type by using the corresponding fraction $f_{\mathrm{p}}$ of Table 2 that set the star number density $n$ in Eq. (3) and hence in $1 / t_{\mathrm{enc}}$. The fractions $f_{\mathrm{p}}$ account for the fact that massive passing stars are rare, but destructive for a disk, and low-mass passing stars are common, but less harmful.

We return to the choice $\phi\left(d<d_{\text {enc }}\right)=6$, which is based on the efficiency with which flybys strip a debris disk depending on the inclination $i$ of the passing star orbit as well as the sense of circulation as discussed in Sect. 2. As already pointed out, a stellar flyby strips a debris disk according to the fractions given in Figs. 2 or 3 only if the passing star and disk motions are prograde and if $i<45^{\circ}$ (two conditions). Assuming that the angular momentum vector of the passing star is randomly distributed over the whole celestial sphere, the differential probability that this vector goes through a celestial band between inclinations $i$ and $i+\mathrm{d} i$ is $\mathrm{d} p=\frac{1}{2} \sin (i) \mathrm{d} i$. By integrating between $i=0$ and $\pi / 4$, the probability corresponding to the two conditions above is $p \sim 1 / 6$. Hence, six flybys must occur to have one that is efficient at stripping the planetesimals of a disk according to the fractions given in Figs. 2 or 3.

Finally, we combine the stripping effects over the six stellar spectral type ranges defined in Table 2 by computing the total fraction of the planetesimals left in the disk after $100 \mathrm{Myr}$, (1 $\left.s_{1}\right) \times\left(1-s_{2}\right) \times \ldots . \times\left(1-s_{6}\right)$, where each $s_{i}$ is the fraction of
Table 3. Closest stellar encounters of the Sun and corresponding fractions of stripped planetesimals ${ }^{(*)}$.

\begin{tabular}{lccc}
\hline \hline Spectral type $m_{\mathrm{p}}$ & $m_{\mathrm{p}} / m_{\mathrm{c}}$ & $\begin{array}{c}d_{\mathrm{enc}} \\
(\mathrm{AU})\end{array}$ & Fraction $s_{i}$ \\
\hline M8-M5 & 0.15 & 180 & 0 \\
M4-M0 & 0.25 & 200 & $2 \%$ \\
K8-K0 & 0.5 & 230 & $3 \%$ \\
G8-G0-F0 & 1.0 & 500 & 0 \\
A8-A0 & 2.0 & 875 & 0 \\
B8-B5 & 4. & 1250 & 0 \\
\hline
\end{tabular}

Notes. ${ }^{(*)}$ Duration $=100 \mathrm{Myr} ; m_{\mathrm{c}}=1 M_{\odot} ; n_{0}=3000 \mathrm{pc}^{-3} ; \sigma=$ $5 \mathrm{~km} \mathrm{~s}^{-1} ; \phi\left(d<d_{\mathrm{enc}}\right)=6$.

stripped planetesimals of Figs. 2 or 3 for the appropriate ratio $m_{\mathrm{p}} / m_{\mathrm{c}}$.

As an example of this calculation, we list in Table 3 the miss distance $d_{\text {enc }}$ and stripping factor $s_{i}$ for each spectral type of the stellar encounters undergone by the Sun for the first $100 \mathrm{Myr}$ after its birth in a cluster characterized by $\sigma=5 \mathrm{~km} \mathrm{~s}^{-1}$ and the initial stellar density $n_{0}=3000 \mathrm{pc}^{-3}$ (Adams \& Laughlin 2001). In these conditions, the total fraction of planetesimals left is $<95 \%$ after $100 \mathrm{Myr}$ in our model. The present day Kuiper belt has only $\sim 1 \%$ of the mass that the minimun mass solar nebula hypothesis predicts, and, the hypothesis of the late heavy bombardement at $700 \mathrm{Myr}$ has been put forth as an explaination (Morbidelli et al. 2005).

In Table 4 , we search the parameter space $\left(m_{\mathrm{c}}, n_{0}\right)$ to find out the conditions in which a debris disk can be most severely depleted during the first $100 \mathrm{Myr}$ of its lifetime. The fractions of planetesimals left after $100 \mathrm{Myr}$ in a standard size disk (40-100 AU) and compact disk (10-40 AU) are estimated for the central star masses, $m_{\mathrm{c}}=0.25,0.5,1.0$ or $2.5 M_{\odot}$, which cover the mass range of stars searched for debris disks in surveys. The cluster dispersion velocity adopted is $\sigma=5 \mathrm{~km} \mathrm{~s}^{-1}$, as observed in 11 nearby open clusters and associations with ages between 5 Myr (Upper Sco) and 757 Myr (Praesepe) by Madsen et al. (2002), and as measured in the $N$-body simulation of the dynamical evolution of embedded clusters from which open clusters emerge (Proszkow \& Adams 2009). In Table 4, the initial star number density $n_{0}$ is chosen to be $100,1000,3000$, 10000,20000 , or $30000 \mathrm{pc}^{-3}$ for several reasons; most embedded clusters have stellar densities of $\sim 100 \mathrm{pc}^{-3}$ (Carpenter 2000, Porras et al. 2003, Lada \& Lada 2003), the Sun is thought to be born in a cluster of stellar density $\sim 3000 \mathrm{pc}^{-3}$ (Adams \& Laughlin 2001), and the densest and closest embedded cluster is the Orion nebula cluster with $\sim 20000 \mathrm{pc}^{-3}$ (Hillenbrand \& Hartmann 1998). We note also that the central stellar density of the Arches cluster close to the Galactic center reaches $\sim 10^{5} \mathrm{pc}^{-3}$ (Portegies Zwart et al. 2007) but we have not included this extreme case in our study.

\section{Discussion}

According to our search of the parameter space $\left(m_{\mathrm{c}}, n_{0}\right)$ in Table 4 , severe depletion by close stellar encounters occurs only for a disk of standard size (40-100 AU) surrounding a star born in an embedded cluster with a high star-density $n_{0}$ greater than $20000 \mathrm{pc}^{-3}$. In these conditions, fewer than $58 \%$ of the planetesimals are left around an intermediate-mass star after 100 Myr, fewer than 37\% around a solar-mass star, and fewer 


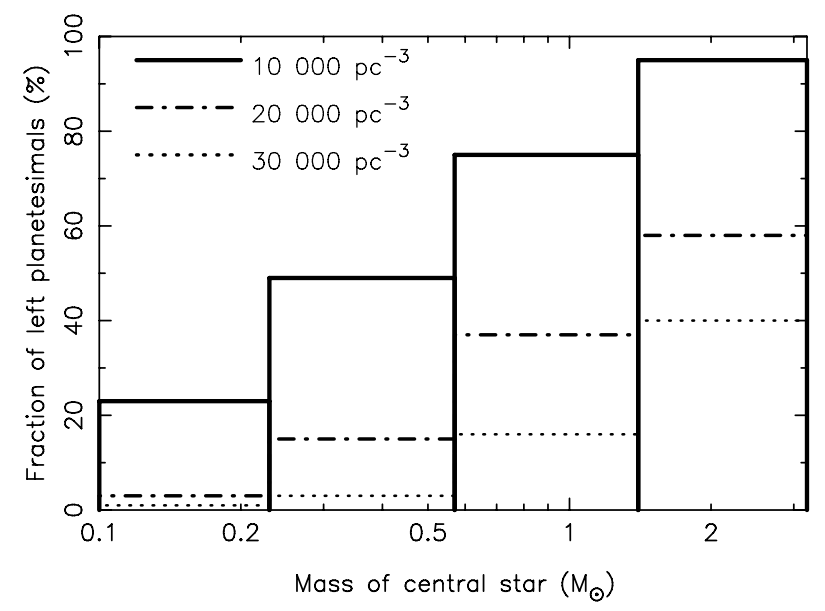

Fig. 4. Fractions of planetesimals left in a debris disk after undergoing close stellar encounters for $100 \mathrm{Myr}$ in open clusters of various initial star number densities. Fractions are the same as in Table 4 for the disk with inner and outer radii of 40 and $100 \mathrm{AU}$. The four bins correspond to the stellar spectral types M8-M5, M4-M0, KGF, and A.

than only $3 \%$ around a low-mass star. In common low stardensity embedded clusters where $n_{0}$ is $<1000 \mathrm{pc}^{-3}$, stellar flybys have a relatively small effect on disks. The turning point in Table 4 where disks start to lose their planetesimals in $100 \mathrm{Myr}$ is the intermediate star density $n_{0} \sim 3000 \mathrm{pc}^{-3}$, which is thought to have prevailed in the birthplace of the Sun. In contrast, disks of compact size (10-40 AU) are almost insensitive to their stellar environment as seen in Table 4. These conclusions remain qualitatively the same regardless of whether the disk is dynamically excited at the start of the simulation or not. In agreement with these results, Spurzem et al. (2009) demonstrated that disruptions of some wide-orbit planetary systems in an Orion-type cluster are expected on a timescale of a few $10^{8}$ yrs, leaving freefloating planets as relics.

We can make a rough estimate of the fraction of stars born in low and high star-density environments by comparing the numbers of stars $N_{1}$ and $N_{2}$ that are, respectively, in the closest high star-density embedded cluster, the Orion nebula cluster at $450 \mathrm{pc}$, and in all low star-density embedded clusters closer than this distance; these numbers are $N_{1}=2520$ and $N_{2}=1324$ based on the catalog of Lada \& Lada (2003). Thus, the corresponding fractions are $N_{2} /\left(N_{1}+N_{2}\right)=34 \%$ and $N_{1} /\left(N_{1}+N_{2}\right)=66 \%$ for stars born in low and high star-density embedded clusters, respectively. If this rough estimate holds over the whole Milky Way, we can conclude that about two-thirds of the stars surveyed today originate from high star-density embedded clusters, thus their disks may have suffered severe stripping by close stellar encounters according to Table 4 . We note that embedded clusters with star-densities higher than $10000 M_{\odot} \mathrm{pc}^{-3}$ survive the initial gas expulsion phase ("infant mortality phase"), and are stable against disruption by stellar evolution and encounters with giant molecular clouds over an Hubble time (Lamers et al. 2005, Gieles 2009).

As depicted in Fig. 4, or equivalently in Table 4, the fraction of planetesimals left in a standard disk (40-100 AU) depends significantly on the mass of the central star $m_{\mathrm{c}}$. This is expected in our model in which flybys are more disruptive for disks around M-dwarfs encountering mostly more massive stars, while they are less damaging for disks around A-dwarfs encountering mostly less massive stars. Consequently, in our model, disks surrounding M-dwarfs are expected to be more difficult to
Table 4. Fractions of planetesimals left in a debris disk after undergoing close stellar encounters for $100 \mathrm{Myr}$ in expanding open clusters of various initial star densities.

\begin{tabular}{|c|c|c|c|}
\hline \multirow{2}{*}{$\begin{array}{c}\text { Central star } \\
m_{\mathrm{c}}\left(M_{\odot}\right)\end{array}$} & \multirow{2}{*}{$\begin{array}{l}\text { Cluster }^{(*)} \\
n_{0}\left(\mathrm{pc}^{-3}\right)\end{array}$} & \multicolumn{2}{|c|}{ Fraction of planetesimals left } \\
\hline & & $\begin{array}{c}\text { Disk } 40-100 \mathrm{AU} \\
(\%)\end{array}$ & $\begin{array}{c}\text { Disk } 10-40 \mathrm{AU} \\
(\%)\end{array}$ \\
\hline 0.25 & 100 & 100 & 100 \\
\hline 0.5 & " & 100 & 100 \\
\hline 1.0 & $"$ & 100 & 100 \\
\hline 2.5 & " & 100 & 100 \\
\hline 0.25 & 1000 & 100 & 100 \\
\hline 0.5 & " & 100 & 100 \\
\hline 1.0 & " & 100 & 100 \\
\hline 2.5 & $"$ & 100 & 100 \\
\hline 0.25 & 3000 & $<65$ & 100 \\
\hline 0.5 & " & $<83$ & 100 \\
\hline 1.0 & " & $<95$ & 100 \\
\hline 2.5 & " & 100 & 100 \\
\hline 0.25 & 10000 & $<23$ & 100 \\
\hline 0.5 & " & $<49$ & 100 \\
\hline 1.0 & " & $<75$ & 100 \\
\hline 2.5 & " & $<94$ & 100 \\
\hline 0.25 & 20000 & $<3$ & $<97$ \\
\hline 0.5 & " & $<15$ & 100 \\
\hline 1.0 & $" \prime$ & $<37$ & 100 \\
\hline 2.5 & $"$ & $<58$ & 100 \\
\hline 0.25 & 30000 & $<1$ & $<76$ \\
\hline 0.5 & " & $<3$ & $<95$ \\
\hline 1.0 & " & $<16$ & 100 \\
\hline 2.5 & " & $<40$ & 100 \\
\hline
\end{tabular}

Notes. ${ }^{(*)} \sigma=5 \mathrm{~km} \mathrm{~s}^{-1}, \quad \phi\left(d<d_{\text {enc }}\right)=6$.

detect since more depleted disks produce less observable dust. This trend in our model agrees with the observed decline in the number of debris disks found around lower mass stars in surveys (32\% for A-dwarfs, 16\% for FGK dwarfs, $<5 \%$ for M-dwarfs) as emphasized in Lestrade et al. (2009).

A limitation of our model is that the star number density is assumed to be uniform across the cluster. Mass segregation is a well-known feature of star clusters, as can be seen in the Trapezium where the most massive stars are arranged in a central, compact core (Moeckel \& Bonnell 2009). Mass segregation can be approximated in our model by a relative increase in the high-mass star fraction $f_{\mathrm{p}}$ given in Table 2 to simulate the condition at the center of the cluster. For example, in the case of $m_{\mathrm{c}}=0.25 M_{\odot}$ and $n_{0}=20000 \mathrm{pc}^{-3}$, after increasing $f_{\mathrm{p}}$ by a factor of four for the most massive stars of this study $\left(m_{\mathrm{p}}=2.5 M_{\odot}\right)$ and decreasing $f_{\mathrm{p}}$ accordingly for the lowest mass stars, the fraction of remaining planetesimals changes from $<3 \%$ in Table 4 to $<2 \%$. For the same modifications of $f_{\mathrm{p}}$ but for $m_{\mathrm{c}}=2.5 M_{\odot}$ and $n_{0}=20000 \mathrm{pc}^{-3}$, the fraction changes from $<58 \%$ in Table 4 to $<45 \%$. Consequently, this limitation of our model makes our estimate conservative and leaves unchanged our main conclusion.

\section{Conclusion}

We have studied the depletion of planetesimals in a debris disk triggered by close stellar encounters in the environment of an 
expanding open cluster over its lifetime of 100 Myr. We have found that depletion is significant only for an initial star-density of the embedded cluster of origin greater than $20000 \mathrm{pc}^{-3}$, as in the Orion nebula cluster, and in a disk of standard size (inner and outer radii : 40 and $100 \mathrm{AU}$ ). In these conditions, a debris disk loses $>97 \%$ of its planetesimals around an M-dwarf, $>63 \%$ around a solar-type star, and $>42 \%$ around an A-dwarf in $100 \mathrm{Myr}$. This level of depletion could affect two-thirds of the stars searched in surveys since two-thirds of them are born in high star-density embedded clusters according to the cata$\log$ of Lada \& Lada (2003). However, more compact disks (inner and outer radii : 10 and $40 \mathrm{AU}$ ) are much less sensitive to their environment. Unfortunately, debris disk sizes are not yet well enough known to decisively conclude whether the observed trend of fewer debris disks being detected around lower mass stars can be explained by the mechanism we have studied.

Acknowledgements. We are grateful to our referee, Hervé Beust, for his suggestions and are indebted to him for indicating the correct probability calculation of the number of flybys. Etienne Morey $\mathrm{PhD}$ work is funded by a Fondation CFM-JP Aguilar grant.

\section{References}

Abt, H. 2009, PASP, 121, 1307

Adams, F. C., \& Laughlin, G. 2001, Icarus, 150, 151

Andrews, S., Wilner, D., Espaillat, C., et al. 2011, ApJ, 732, 42

Augereau, J.-C., \& Beust, H. 2006, A\&A, 455, 987

Augereau, J.-C., Nelson, R. P., Lagrange, A.-M., Papaloizou, J. C. B., \& Mouillet, D. 2001, A\&A, 370, 447

Beust, H., \& Dutrey, A. 2006, A\&A, 446, 137

Binney, J., \& Tremaine, S. 1987, Galactic Dynamics (Princeton Unversity Press) Bryden, G. C., Beichman, C. A., Trilling, D. E., et al. 2006, ApJ, 636, 1098

Bonnell, I. A., Kester, W. S., Melvyn, B. D., \& Keith, H. 2001, MNRAS, 322, 859

Booth, M., Wyatt, M. C., Mobidelli, A., Moro-Martin, A., \& Levison, H. F. 2010, MNRAS, 399, 385

Carpenter, J. 2000, AJ, 120, 3139

Converse, J. M., \& Stahler, S. W. 2010, MNRAS, 405, 666

Corder, S., Carpenter, J. M., Sargent, A. I., et al. 2009, ApJ, 690, L65

Dent, W. R. F., Walker, H. J., Holland, W. S., \& Greaves, J. S. 2000, MNRAS, 314,702

Dominik, C., \& Decin, G. 2003, ApJ, 583, 626.

Eiroa, C., Fedele, D., Maldonato, J., et al. 2010, A\&A, 518, L131
Garcia-Sanchez, J., Weissman, P. R., \& Preston, R. A. 2001, A\&A, 379, 634

Gautier, T. N., Rieke, G. H., Stansberry, J., et al. 2007, ApJ, 667, 527

Gieles, M. 2009, in Star Clusters, Proceedings of the ed. R. de Grijs, \& J.R.D.

Lépine (Cambridge University Press), IAU Symp., 266

Gomes, R., Levison, H. F., Tsiganis, K., \& Morbidelli, A. 2005, Nature, 435, 466

Greaves, J. S., Holland, W. S., Wyatt, M. C., et al. 2005, ApJ, 619, 187

Hillenbrand, L., \& Hartmann, L. W. 1998, ApJ, 492, 540

Kaib, N. A., \& Quinn, T. 2008, Icarus, 197, 221

Kalas, P., Larwood, J., Smith, B. A., \& Schultz, A. 2000, ApJ, 530, L133.

Kennedy, G. M., \& Wyatt, M. C. 2010, MNRAS, 405, 1253

Kenyon, S. J., \& Bromley, B. C. 2002, ApJ, 577, L35

Kenyon, S. J., \& Bromley, B. C. 2004, Nat., 432, 598

Kobayashi, H., \& Ida, S. 2001, Icarus, 153, 416

Lada, C. J., \& Lada, E. A. 2003, ARA\&A, 41, 57

Lamers, H. J. G. L. M., Gieles, M., Bastian, N., et al. 2005, A\&A, 441, 117

Larwood, J. D. 1997, MNRAS, 290, 490

Larwood, J. D., \& Kalas, P. G. 2001, MNRAS, 323, 402

Lestrade, J.-F., Wyatt, M. C., Bertoldi, F., Dent, W. R. F., \& Menten, K. M. 2006, A\&A, 460, 733

Lestrade, J.-F., Wyatt, M. C., Bertoldi, F., Menten, K. M., \& Labaigt, G. 2009, A\&A, 506, 1455

Liseau, R., Risacher, C., Brandeker, A., et al. 2008, A\&A, 480, L47

Madsen, S., Dravins, D., \& Lindegren, L. 2002, A\&A, 381, 446

Malmberg, D., de Angeli, F., Davies, M. B., et al. 2007, MNRAS, 378, 1207

Marshall, J. P., Löhne, T., Montesinos, B., et al. 2011, A\&A, 529, 117

Matthews, B. C., Sibthorpe, B., Kennedy, G., et al. 2010, A\&A, 518, L135

Moeckel, N., \& Bonnell, I. A. 2009, MNRAS, 400, 657

Morbidelli, A., Levison, H. F., Tsiganis, K., \& Gomes, R. 2005, Nature, 435, 462

Moro-Martín, A., \& Malhotra, R. 2002, AJ, 124, 2305

Mouillet, D., Larwood, J. D., Papaloizou, J. C. B, \& Lagrange, A.-M. 1997, MNRAS, 292, 896

Mustill, A., \& Wyatt, M. C. 2009, MNRAS, 399, 1403

Najita, J., \& Williams, J. P. 2005, ApJ, 635, 625

Olczack, C., Pfalzner, S., \& Eckart, A. 2009, A\&A, 509, 630

Porras, A., Christopher, M., Allen, L., et al. 2003, AJ, 126, 1916

Portegies Zwart, S., Gaburov, E., Chen, H.-C., \& Gürkan, M. A. 2007, MNRAS, 378, L29

Press, W. H., Teukolsky, S. A., Vetterling, W. T., \& Flannery, B. P. 1992 (Cambridge University Press)

Proszkow, E.-M., \& Adams, F. C. 2009, ApJS, 185, 486

Reche, R., Beust, H., \& Augereau, J.-C. 2009, A\&A, 493, 661

Spurzem, R., Giersz, M, Heggie, D. C., \& Lin, D. N. C. 2009, ApJ, 697, 458

Su, K. Y. L., Rieke, G. H., Stapelfeldt, K. R., et al. 2006, ApJ, 653, 675

Toomre, A., \& Toomre, J. 1972, ApJ, 178, 623

Trilling, D. E., Bryden, G., Beichman, C. A., et al. 2008, ApJ, 674, 1086

Tsiganis, K., Gomes, R., Morbidelli, A., \& Levison, H. F. 2005, Nature, 435, 459

Wyatt, M. C. 2003, ApJ, 598, 1321

Wyatt, M. C. 2008, ARA\&A, 46, 339

Wyatt, M. C., \& Dent, W. F. R., \& Greaves, J. S. 2003, MNRAS, 342, 876 